\title{
Primeiros estudos citotaxonômicos e distribuição geográfica de Rhynchosia naineckensis Fortunato (Leguminosae) para o estado de Goiás
}

\author{
Elaine Biondo ${ }^{1}$ \\ André RosalvoTerra Nascimento ${ }^{3}$ \\ Sílvia Teresinha Sfoggia Miotto ${ }^{1}$ \\ Maria Teresa Schifino-Wittmann ${ }^{2}$
}

\begin{abstract}
RESUMO
O objetivo deste trabalho foi determinar o número cromossômico e citar nova ocorrência na distribuição geográfica de Rhynchosia naineckensis Fortunato para o estado de Goiás. Foram coletados espécimes em três diferentes populações naturais ocorrentes em fragmentos de floresta decídua, localizados na região nordeste de Goiás, Brasil. A espécie é diplóide com $2 n=2 x=22$ cromossomos. Foram observados cromossomos metacêntricos e submetacêntricos e núcleos interfásicos arreticulados. Rhynchosia naineckensis está sendo citada como primeira ocorrência para o estado de Goiás.
\end{abstract}

Palavras-chave: Rhynchosia, cromossomos, núcleo interfásico, distribuição, Leguminosae

\begin{abstract}
This work aimed to determine the chromosome number and report a new occurrence in the geographical distribution of Rhynchosia naineckensis Fortunato to Goias state. Specimen were collected from three different natural populations from fragments of deciduous forest in Northeast Goiás. This species is diploid, with $2 \mathrm{n}=2 \mathrm{x}=22$ chromosomes. Meta and submetacentric chromosomes and arreticulate interphase nuclei were observed. This is the first citation of Rhynchosia naineckensis for the state of Goiás.
\end{abstract}

Keywords: Rhynchosia, chromosomes, interphase nuclei, distribution, Leguminosae

\section{INTRODUÇÃO}

O gênero Rhynchosia Lour. pertence à subtribo Cajanineae, tribo Phaseoleae (Leguminosae - Papilionoideae) e apresenta espécies distribuídas pelas regiões tropicais e subtropicais de ambos os hemisférios (Grear, 1978; Fortunato, 1982, 1999a; Burkart, 1987; Bianco \& Kraus, 1995). Este gênero pantropical compreende um número aproximado de 200 espécies na África (Grear, 1978; Burkart,
1987) e 57 espécies no Novo Mundo, com distribuição na América do Sul, América Central e Caribe (Fortunato, 1983, 1999a, 2000). Para o Brasil foram citadas 18 espécies (Grear, 1978).

Duke (1959), realizando estudos botânicos no estado do Ceará, citou duas espécies de Rhynchosia. Angely (1970), em um levantamento florístico para o estado de São Paulo, citou nove espécies. Miotto (1988)

\footnotetext{
${ }_{1}^{1}$ Programa de Pós-graduação em Botânica, Universidade Federal do Rio Grande do Sul, Porto Alegre, RS.

2 Programa de Pós-graduação em Zootecnia, Universidade Federal do Rio Grande do Sul, Porto Alegre, RS.

${ }^{3}$ Programa de Pós-graduação em Ecologia, Universidade de Brasília, Brasília, DF.

Apoio Financeiro: CAPES, CNPq.

Endereço para correspondência: Elaine Biondo, Rua João Telles, 453/32, B. Bom Fim, Porto Alegre, Rio Grande do Sul, Brasil. CEP 90035-121. elainebiondo@ hotmail.com
} 
citou para o Rio Grande do Sul, nove espécies. Lewis (1987), estudando as leguminosas da Bahia, citou cinco espécies e duas variedades do gênero. Mais recentemente, foram realizados levantamentos da Flora do Cerrado (UNESCO, 2000) e, entre as espécies listadas para a Reserva da Biosfera do Cerrado - Fase I, foram descritas duas espécies de Rhynchosia. Proença et al. (2001) citaram para a flora do Distrito Federal, oito espécies do gênero. Felfili \& Silva Jr. (2001) citaram uma espécie deste gênero para a caatinga na Bahia.

O estudo taxonômico mais abrangente do gênero foi realizado por Grear (1978), que revisou as espécies de Rhynchosia do Novo Mundo.

As espécies do gênero Rhynchosia ocorrentes no Novo Mundo foram incluídas em três seções: Cyanospermum, Copisma, dividida em quatro séries, eArcyphyllum com duas séries. Todas as seções possuem representantes com distribuição no Brasil, com exceção da seção Cyanospermum (Grear, 1978). Características morfológicas de partes vegetativas e reprodutivas de espécies do gênero Rhynchosia foram relacionadas e analisadas por Fortunato (1999a), e revelaram evidências para a circunscrição da seção Arcyphyllum, para descrever duas novas séries dentro da seção Copisma e reconhecer a seção Phaseoloides Benth. emend Fortunato.

Na série 2, da seção Copisma, cujas sementes são bicolores (vermelho e preto), estão incluídas sete espécies: $R$. melanocarpa Grear, $R$. pyramidalis (Lam.) Urban, $R$. erythrinoides Cham. \& Schltdl., $R$. quercetorum Standl., R. phaseoloides (Sw.) DC., $R$. amabilis Grear e $R$. precatoria (Humb. \& Bonpl. ex Willd.) DC. (Grear, 1978). Fortunato (1982) descreveu Rhynchosia naineckensis incluindo-a nesta seção e série.

Rhynchosia naineckensis é uma liana com folhas pinado-trifolioladas e folíolos deltóides, apresentando legumes contraídos entre as sementes bicolores, pretas e com uma área vermelha ao redor do hilo. A espécie é próxima de Rhynchosia melanocarpa e de Rhynchosia phaseoloides, podendo distinguir-se pelo padrão de distribuição das cores nas sementes, presença ou ausência de estipelas, tamanho das brácteas e do pedicelo (Fortunato, 1982). Distribui-se pelo Paraguai, montes altos do norte Argentino, sul da Bolívia e no Brasil (Fortunato, 1999b). No Brasil, ocorre no Mato Grosso e São Paulo (Fortunato, com. pes.).

Análises de número e morfologia de cromossomos, comportamento meiótico e reprodutivo podem ser utilizados em estudos biossistemáticos, contribuindo também para o entendimento taxonômico e evolutivo dos grupos, sendo indispensáveis em cruzamentos programados e para a inclusão de espécies em bancos de germoplasma (Valls, 1988; Schifino-Wittmann, 2000). O número cromossômico normalmente é constante dentro da espécie, embora possam ocorrer variações como aneuploidias e poliploidias, entre os indivíduos e populações com diferente distribuição geográfica (Stebbins, 1971).

Entre as espécies de Rhynchosia são verificados números cromossômicos de $2 \mathrm{n}=$ 20, 22 e 24 , sendo $2 n=22$ o mais comum, com apenas duas espécies citadas com $2 \mathrm{n}=$ 24 e uma com $2 \mathrm{n}=20$ (Federov, 1969; Goldblatt, 1981 a; Goldblatt, 1981b, 1984, 1985, 1988; Goldblatt \& Johnson, 1990, 1991, 1994, 1998) sendo este, portanto, um gênero relativamente conservador para o número cromossômico.

Estudos cromossômicos são escassos nas espécies do gênero Rhynchosia ocorrentes no Brasil. Análises de número cromossômico foram realizadas por Biondo (2000) em quatro espécies do gênero Rhynchosia ocorrentes na região Sul do Brasil, sendo que $R$. corylifolia Mart. ex Benth., $R$. hauthalii (O. Kuntze) Grear, $R$. diversifolia M. Micheli var. diversifolia possuem $2 \mathrm{n}=22$ e R. edulis Griseb. $2 \mathrm{n}=20$.

Face ao exposto, este trabalho teve como 
Primeiros estudos citotaxonômicos e distribuição geográfica de Rhynchosia naineckensis Fortunato

objetivos realizar as primeiras contagens cromossômicas em Rhynchosia naineckensis e citar nova ocorrência geográfica da espécie para o estado de Goiás.

\section{MATERIAL E MÉTODOS}

As sementes foram coletadas em populações de $R$. naineckensis em três diferentes fragmentos de floresta decídua no nordeste do estado de Goiás. As exsicatas coletadas estão depositadas nos Herbários ICN ou CEN (Tabela 1).

As contagens cromossômicas foram realizadas no Laboratório de Citogenética e Eletroforese - Departamento de Plantas Forrageiras e Agrometeorologia - UFRGS/ RS. As sementes foram escarificadas e colocadas para germinar em placas de petri, em germinador com fotoperíodo controlado com temperatura de $25^{\circ} \mathrm{C}$. As radículas foram pré-tratadas em para-dicloro-benzeno, por 18$20 \mathrm{~h}, \mathrm{a} 4^{\circ} \mathrm{C}$ e fixadas com Carnoy por $24 \mathrm{~h}$, em temperatura ambiente. A hidrólise foi realizada com $\mathrm{HCL} 1 \mathrm{~N}$, por $10 \mathrm{~min}, \mathrm{a} 60^{\circ} \mathrm{C} \mathrm{e}$ o corante utilizado foi Fuelgen. Foram contadas e fotografadas dez células metafásicas para cada população. O padrão de condensação de núcleo interfásico também foi analisado, tendo sido observados 200 núcleos celulares para cada população, a classificação do padrão de condensação do núcleo interfásico seguiu Guerra (1985).

Tabela 1 - Local de coleta, coletor, número do coletor e sigla dos herbários onde estão depositadas as exsicatas provenientes de três diferentes populações de Rhynchosia naineckensis Fortunato.

\begin{tabular}{lll}
\hline & Local de Coleta & Coletor / no/ Herbário \\
\hline POP 1 & São Domingos/GO & E. Biondo, 001 (ICN) \\
POP 2 & São Domingos/GO & A. R. T. Nascimento, \\
& & $220(\mathrm{CEN})$ \\
POP 3 São Domingos/GO & A. R. T. Nascimento, \\
& & $221(\mathrm{CEN})$ \\
\hline
\end{tabular}

Rodriguésia 54 (83): 5-11. 2003
Com o objetivo de esclarecer a distribuição de $\mathrm{R}$. naineckensis no estado de Goiás, foram feitos levantamentos nos seguintes herbários: CEN, HUEFS, ICN, INPA, SMDB, UB (siglas segundo Holmgren et al., 1990).

\section{RESULTADOS E DISCUSSÃO}

As características citológicas mais comumente utilizadas em estudos taxonômicos são o número de cromossomos, seu tamanho e forma, padrão de condensação profásico e de núcleos interfásicos, conteúdo de DNA e comportamento meiótico (Stuessy, 1990). Todos os espécimes de $R$. naineckensis analisados, apresentaram número cromossômico diplóide $2 \mathrm{n}=2 \mathrm{x}=22$ (Fig. 1). Foramobservados cromossomos metacêntricos e submetacêntricos, variando de 1 a $3 \mathrm{~mm}$, aproximadamente. A análise de condensação de núcleo interfásico mostrou padrão de núcleo interfásico arreticulado (Fig. 1).

$R$. naineckensis possui número básico de cromossomos $\mathrm{n}=\mathrm{x}=11$, como a grande maioria das espécies deste gênero. Biondo (2000) descreveu para quatro espécies do gênero Rhynchosia, ocorrentes na região Sul do Brasil, cariótipos com tendências simétricas, com cromossomos metacêntricos e submetacêntricos. $\mathrm{O}$ número básico foi $\mathrm{n}=\mathrm{x}$ $=11$ cromossomos e apenas $R$. edulis apresentou $\mathrm{n}=\mathrm{x}=10$ cromossomos. Goldblatt (1981a) considerou $\mathrm{x}=11$ o número básico predominante no gênero Rhynchosia, assim como na maioria dos gêneros da tribo Phaseoleae. Os dados de literatura mostram que 25 espécies possuem $2 \mathrm{n}=22$ cromossomos (Federov, 1969; Goldblatt, 1981b, 1984, 1985; Goldblatt \& Johnson, 1990, 1991, 1994, 1998, Biondo, 2000) (Tabela 2), R. edulis apresenta $2 \mathrm{n}=20$ (Biondo, 2000) e somente duas espécies possuem $2 \mathrm{n}=24$ cromossomos, $R$. pyramidalis (Goldblatt, 1981 b) e $R$. pycnostachya (Federov, 1969; Goldblatt, 1988, Goldblatt \& Johnson, 1990). Comparando-se o número de cromossomos das espécies da Secção Copisma, Série 2, 


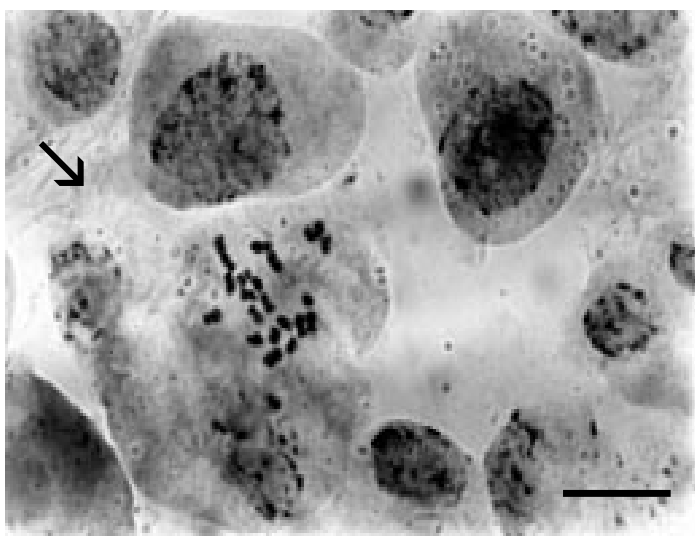

Figura 1 - Metáfase mitótica com $2 n=2 x=22$ cromossomos e núcleo interfásico com padrão de condensação arreticulado (seta) em Rhynchosia naineckensis Fortunato. Barra $=10 \mu \mathrm{m}$. observou-se que somente $R$. pyramidalis possui número cromossômico $2 \mathrm{n}=24$ (Goldblatt, 1981b), sendo que as demais espécies possuem $2 \mathrm{n}=22$ cromossomos.

As variações no número cromossômico observadas no gênero provavelmente ocorreram por aneuploidia ou por rearranjos cromossômicos ocorridos no decorrer do processo evolutivo do mesmo.

Existem algumas tendências citológicas comuns em muitas famílias e gêneros, características como núcleo interfásico reticulado e cariótipos simétricos, são mais freqüentemente observados em representantes primitivos, sendo que tendências opostas a

Tabela 2 - Contagens prévias do número de cromossomos em espécies do gênero Rhynchosia Lour

\begin{tabular}{|c|c|c|}
\hline Espécies & $\begin{array}{l}\text { No de } \\
\text { cromossomos 2n }\end{array}$ & Autores trabalhos anteriores \\
\hline Rhynchosia minima (L.) DC. & 22 & $\begin{array}{l}\text { Federov, 1969; Goldblatt, 1981b, 1984, 1988; Goldblatt } \\
\text { \& Johnson, 1991, 1994, } 1998\end{array}$ \\
\hline R. rufescens (Willd.) DC. & 22 & Goldblatt \& Johnson, 1991 \\
\hline R. capitata (Heine ex Roth) DC. & 22 & Federov, 1969; Goldblatt, 1984, 1988; Goldblatt \& Johnson, 1994 \\
\hline R. phaseoloides DC. & 22 & Federov, 1969; Goldblatt, 1981b, 1984 \\
\hline R. reticulata $($ Swartz) DC. & 22 & Goldblatt, 1984 \\
\hline R. sublobata (Schumach.) Meikle & 22 & Goldblatt, $1981 b$ \\
\hline R. bracteata Benth ex. Baker & 22 & Goldblatt, 1981b, Goldblatt \& Johnson, 1994R. diversifolia \\
\hline M. Mich. & 22 & Goldblatt, $1981 b$ \\
\hline R. rothii Benth ex.Aitchison & 22 & Goldblatt, 1981b \\
\hline R. sericea Spanogue & 22 & Goldblatt, 1981b, Goldblatt \& Johnson, 1994 \\
\hline R. puberulenta Stocks & 22 & Goldblatt \& Johnson, 1994, 1998 \\
\hline R. suaveolens (L.f.) DC. & 22 & Goldblatt \& Johnson, 1998 \\
\hline R. volubilis Lour. & 22 & Federov, 1969; Goldblatt, 1988; Goldblatt \& Johnson, 1998 \\
\hline R. texana Torr et. A. Gray & 22 & Federov, 1969; Goldblatt, 1988 \\
\hline R. hirta (Andr.) Meikle \& Verdc. & 22 & Goldblatt, 1985 \\
\hline R. americana (Mill.) Metz & 22 & Federov, 1969 \\
\hline R. aurea DC. & 22 & Federov, 1969 \\
\hline R. debilis Hook. f. & 22 & Federov, 1969 \\
\hline R. difformis (Ell.)DC. & 22 & Federov, 1969 \\
\hline$R$. erecta (Walt.) DC. & 22 & Federov, 1969 \\
\hline R. himalensis Benth. & 22 & Federov, 1969 \\
\hline R. latifolia Nutt. & 22 & Federov, 1969 \\
\hline R. senna Gillies ex. Hooker & 22 & Federov, 1969 \\
\hline R. corylifolia Martius ex. Benth & 22 & Biondo 2000 \\
\hline R. hauthalii (O.Kuntz) Grear & 22 & Biondo 2000 \\
\hline R. edulis Griseb. & 20 & Biondo 2000 \\
\hline R. pycnostachya (DC.) Meikle & 24 & Federov, 1969; Goldblatt, 1988; Goldblatt \& Johnson, 1990 \\
\hline R. pyramidalis (Lamark) Urban & 24 & Goldblatt, 1981b \\
\hline R. naineckensis Fortunato & 22 & Presente trabalho \\
\hline
\end{tabular}


Primeiros estudos citotaxonômicos e distribuição geográfica de Rhynchosia naineckensis Fortunato

estas são verdadeiras, com diversos casos documentados (Guerra, 1999). Entretanto, como o conteúdo de informações citogenéticas ainda é incipiente em espécies brasileiras do gênero Rhynchosia, uma discussão a respeito ainda é prematura.

Está sendo registrada a nova ocorrência geográfica de $R$. naineckensis para o nordeste do estado de Goiás. Até o momento, esta espécie foi coletada em três diferentes fragmentos de Floresta Estacional Decidual, incluída no Bioma Cerrado.

$\mathrm{Na}$ compilação da flora vascular do Cerrado, foram citadas sete espécies para o gênero Rhynchosia, não tendo sido registrada a ocorrência de $R$. naineckensis (Mendonça et al., 1998).

No levantamento florístico do município de Alto Paraíso, Goiás, Munhoz \& Proença (1998) citaram a ocorrência de somente uma espécie, $R$. platyphylla Benth., considerada nova citação para a flora do Cerrado.

Proença et al. (2001) citaram para a flora do Distrito Federal oito espécies de Rhynchosia, estando a maior parte delas em ambiente de florestas de galeria.

Entre as espécies listadas pela UNESCO (2000), estão $R$. edulis e $R$. melanocarpa, dentre as leguminosas prioritárias para recuperação da vegetação do Bioma Cerrado.

Felfili \& Silva Jr. (2001), realizando levantamentos florísticos no Espigão Mestre do São Francisco até a Bahia, citaram uma espécie coletada em vegetação de caatinga: $R$. phaseoloides.

De acordo com o exposto acima, ainda são escassos os estudos sobre distribuição geográfica e citogenética das espécies do gênero Rhynchosia em diversas regiões brasileiras, evidenciando-se a necessidade de se intensificar as coletas e os estudos biossistemáticos das mesmas.

\section{CONCLUSÕES}

O número básico cromossômico proposto pela primeira vez para $R$. naineckensis é $\mathrm{n}=$ 11 cromossomos, e os cromossomos desta espécie são metacêntricos e submetacêntricos com núcleos interfásicos arreticulados. Para que sejam possíveis discussão de características citogenéticas e questões evolutivas em Rhynchosia, há necessidade de ampliação das análises.

A ocorrência de $R$. naineckensis está sendo registrada pela primeira vez para o estado de Goiás.

\section{AGRADECIMENTOS}

À EMBRAPA/CENARGEN pelo apoio logístico e possibilidade de coleta das exsicatas a das sementes. À Renée H. Fortunato pela confirmação da identificação e pelas informações sobre Rhynchosia naineckensis. A Bruno Walter e Glocimar P. Silva pela discussão sobre a espécie. Aos senhores Lincon Pereira e José Pereira pela permissão de trabalhar em suas propriedades.

\section{REFERÊNCIAS BIBLIOGRÁFICAS}

Angely, J. 1970. Flora analítica $e$ fitogeográfica do estado de São Paulo. São Paulo: Universidade de São Paulo. p. 285-286.

Bianco, C.A. \& Kraus, T.A. 1995. El genero Rhynchosia en el sur de la Provincia de Cordoba. Rev. Fac. Agronomia 8(1):2939.

Biondo, E. 2000. Citogenética e valor nutritivo em espécies de Rhynchosia Lour. e Eriosema (DC.) G. Don (Leguminosae) nativas na região sul do Brasil. Santa Maria, Universidade Federal de Santa Maria. 87p. 10 il.

Burkart, A. 1987. Leguminosae. In: Burkart, N.S.T. de \& Bacigalupo, N.M. (eds.) Flora Ilustrada de Entre Rios (Argentina). Buenos Aires. 6(3):695 704.

Duke, A. 1959. Estudos botânicos no Ceará. Anais da Academia Brasileira de Ciências 31(2): 211-308.

Federov, A.A. 1969. Chromosome numbers of flowering plants. Leningrado: Russian Academy of Sciences. 
Felfili, J.M. \& Silva Júnior, M.C. (orgs.) 2001. Biogeografia do Bioma Cerrado: estudo fitofisionômico na chapada do Espigão Mestre do São Franscisco. Brasília: Universidade de Brasília, Faculdade de Tecnologia, Departamento de Engenharia Florestal. 152p.

Fortunato, R.H. 1982. Una nueva especie del género Rhynchosia (Leguminosae). Darwiniana 24(1-4): 497-501.

1983. Sinopsis de las espécies Argentinas del genero Rhynchosia nom. cons. Parodiana 2(1):25-58.

. 1999a. Systematic relationship in Rhynchosia (Cajaninae-PhaseoleaePapilionoideae-Fabaceae) from neotropics regions. In: XVI Internacional Botanical Congress. Abstract number 4329.

1999b. Fabaceae. In. Zuloaga, F.O. \& Morrone, O. (eds.). Catálogo de las Plantas Vasculares de la República Argentina II. Sant Louis, Missouri, Missouri Botanical Garden Press, vol. 74, $726 \mathrm{p}$. . 2000. Systematic relationships in Rhynchosia (Cajaninae-PhaseoleaePapilionoideae-Leguminosae) from the neotropics. In. Herendeen, P.S. \& Bruneau, A. (eds.). Advances in Legume Systematics. The Royal Botanic Gardens, Kew, vol. 9, p. 339-354.

Grear, J.W. 1978. A Revision of the New World Species Rhynchosia (Leguminosae - Faboideae). Memoirs of the New York Botanical Garden 31(1):1-168.

Goldblat, P. 1981a. Cytology and the phylogeny of Leguminosae. In.: Polhill, R.M. \& Raven, P.H.(eds). Advances in Legume Systematic, Royal Botanic Gardens, Kew, vol. 2, p. 427-463.

. 1981b. Index to plant chromosome numbers 1975-1978. Monographs in Systematic Botany 5. Missouri Botanical Garden. 1984. Index to plant chromosome numbers 1979-1981. Monographs in Systematic Botany 8. Missouri Botanical Garden.

. 1985. Index to plant chromosome numbers 1982-1983. Monographs in Systematic Botany 13. Missouri Botanical Garden.

. 1988. Index to plant chromosome numbers 1984-1985. Monographs in Systematic Botany 23. Missouri Botanical Garden.

Goldblat, P. \& Johnson, D.E. 1990. Index to plant chromosome numbers 19861987. Monographs in Systematic Botany 30. Missouri Botanical Garden.

1991. Index to plant chromosome numbers 1988-1989. Monographs in Systematic Botany 40. Missouri Botanical Garden.

1994. Index to plant chromosome numbers 1990-1991. Monographs in Systematic Botany 51. Missouri Botanical Garden.

1998. Index to plant chromosome numbers 1994-1995. Monographs in Systematic Botany 69. Missouri Botanical Garden.

Guerra, M.S. 1985. Estrutura e diversificação dos núcleos interfásicos em plantas. In.: I Colóquio sobre Citogenética $e$ Evolução de Plantas, Piracicaba, p. 137-153.

. 1999. A identificação de cariótipos ancestrais em plantas. Genetics and Molecular Biology 22(3):5.

Holmgren, P.K.; Holmgren, N.H.; Barnett, LC. 1990. Index Herbariorum. 8. ed. New York: New York Botanical Garden. 691p. Lewis, G. P. 1987. Legumes da Bahia. Royal Botanic Garden. Kew. 369p.

Mendonça, R.C., Felfili, J.M., Walter, B.M.T., Silva Júnior, M.C., Rezende, A.V.; Filgueiras, T.S.; Nogueira, P.E. 1998. Flora Vascular do Cerrado. In: Sano, S.M.; Almeida, S.P. de (ed.) Cerrado: ambiente e flora. Planaltina: EMBRAPA-CPAC. p. 289-556. 
Primeiros estudos citotaxonômicos e distribuição geográfica de Rhynchosia naineckensis Fortunato

(Leguminosae) para o estado de Goiás

Miotto, S.T.S., 1988. Leguminosae Faboideae. Tribo Phaseoleae - Subtribo Cajaninae. Flora Ilustrada do Rio Grande do Sul 19, B. Inst. Bioc. 43:188.

Munhoz, C.R.; Proença, C.E.B. 1998. Composição florística do município de Alto Paraíso de Goiás na Chapada dos Veadeiros. Boletim do Herbário Ezequias Paulo Heringer 3:102-150.

Proença, C.E.B.; Munhoz, C.B.R.; Jorge, C.L.; Nóbrega, M.G.G. 2001. Listagem e nível de proteção de fanerógamas do Distrito Federal, Brasil. In. Cavalcanti, T.B. \& Ramos, A.E. (orgs.) Flora do Distrito Federal. Brasília: Embrapa Recursos Genéticos e Biotecnologia. p. 89-359.

Schifino-Wittmann, M.T. 2000. The cytogenetics and evolution of forage legumes from Rio Grande do Sul: a review. Genetics and Molecular Biology 23 (4): 989-995.

Stebbins, G.L. 1971. Chromosomal Evolution in Higher Plants. Massachusetts, Addison-Wesley Publishing Company. 216p.

Stuessy, T.F. 1990. Plant Taxonomy: the systematic evolution of comparative data. New York, Columbia University Press., 514 p.

UNESCO. 2000. Vegetação no Distrito Federal - tempo e espaço. Brasília: UNESCO. 74p.

Valls, J.F.M. 1988. Caracterização morfológica, reprodutiva e bioquímica de germoplasma vegetal. In. Araújo, S.M.C. \& Osuna, J.A. (eds.) Encontro sobre recursos genéticos. Jaboticabal, Faculdade de Ciências Agrárias e Veterinárias, UNESP, p.106-128. 